Supporting Information

\title{
Highly conductive doped hybrid carbon nanotube-graphene wires
}

Sandra Lepak-Kuc, ${ }^{\dagger}$ Karolina Z. Milowska,,$+\ddagger$ Slawomir Boncel, Miroslaw Szybowicz, ${ }^{\S}$ Anna Dychalska, ${ }^{\S}$ Iwona Jozwik,,$\perp$ Krzysztof K. Koziol,\# Malgorzata Jakubowska, ${ }^{\dagger}$ and Agnieszka Lekawa-Raus*, ${ }^{*}$

$\dagger$ Department of Mechatronics, Warsaw University of Technology, Poland $\ddagger T C M$ Group, Cavendish Laboratory, University of Cambridge, United Kingdom \Faculty of Chemistry, Silesian University of Technology, Poland $\S$ Faculty of Technical Physics, Poznan University of Technology, Poland || Institute of Electronic Materials Technology, Poland $\perp$ National Centre for Nuclear Research/NOMATEN Centre of Excellence, Poland \#Enhanced Composites \& Structures Centre, Cranfield University, United Kingdom

E-mail: kzm21@cam.ac.uk; alekawa@mchtr.pw.edu.pl 


\section{S1. Structural Properties}

The results presented in this section correspond to the equilibrium situation at $0 \mathrm{~K}$ in vacuum. The optimized geometries of the hybrid systems are bases for calculations of their electronic and transport properties.

The distortion in the carbon network caused by the functionalization of the CNTs $+\mathrm{G}$ hybrid systems is quantified using the coefficient of variation of the CNT radius, CV, which is defined as the ratio of the standard deviation to the mean of the CNT radius. ${ }^{1}$

\section{S2. Electronic and Transport Properties}

The transmission coefficients, $\mathrm{T}(\epsilon, \mathrm{U})$, of electrons incident at energy $\epsilon$ through the central scattering region under a bias voltage $U=\frac{1}{e}\left(\mu_{l}-\mu_{r}\right)$, was calculated using the following expression:

$$
T(\epsilon, U)=\left[\Gamma_{l}(\epsilon) G^{r}(\epsilon) \Gamma_{r}(\epsilon) G^{a}(\epsilon)\right]
$$

where $G^{r(a)}(\epsilon)$ retarded (advanced) Green's function of the central region, $\Gamma_{l(r)}$ is a matrix accounting for the coupling of the central region to the right (left) electrode, and $\mu_{l(r)}$ is the electrochemical potential of the left (right) electrode. Hence, the conductance can be expressed as follows: ${ }^{2}$

$$
G=G_{o} \operatorname{Tr}[T(\epsilon, U)]
$$

where $G_{o}=2 e^{2} / h$ is the unit of quantum conductance. Consequently, the specific conductance $G_{s}$ is expressed as $\mathrm{G} / \mathrm{m}$, where $\mathrm{m}$ is a molecular weight of the central part of the device system.

The electrical current through the device under non-equilibrium conditions was calculated using the Landauer-Büttiker formula:

$$
I(U)=\frac{2 e}{h} \int_{-\infty}^{+\infty} T(\epsilon, U)\left(f_{l}\left(\epsilon-\mu_{l}\right)-f_{r}\left(\epsilon-\mu_{r}\right)\right) d \epsilon
$$


where $f_{(l(r))}$ is the corresponding electron distribution.

Electron transport is assumed to be in the ballistic regime where charge carriers can travel across the device configuration without scattering. Note that inelastic effects are not taken into account in this method.

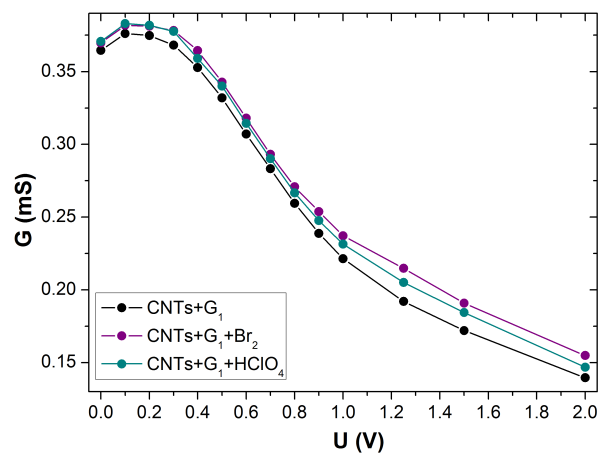

Figure S1: The computed conductance as a function of applied voltage.
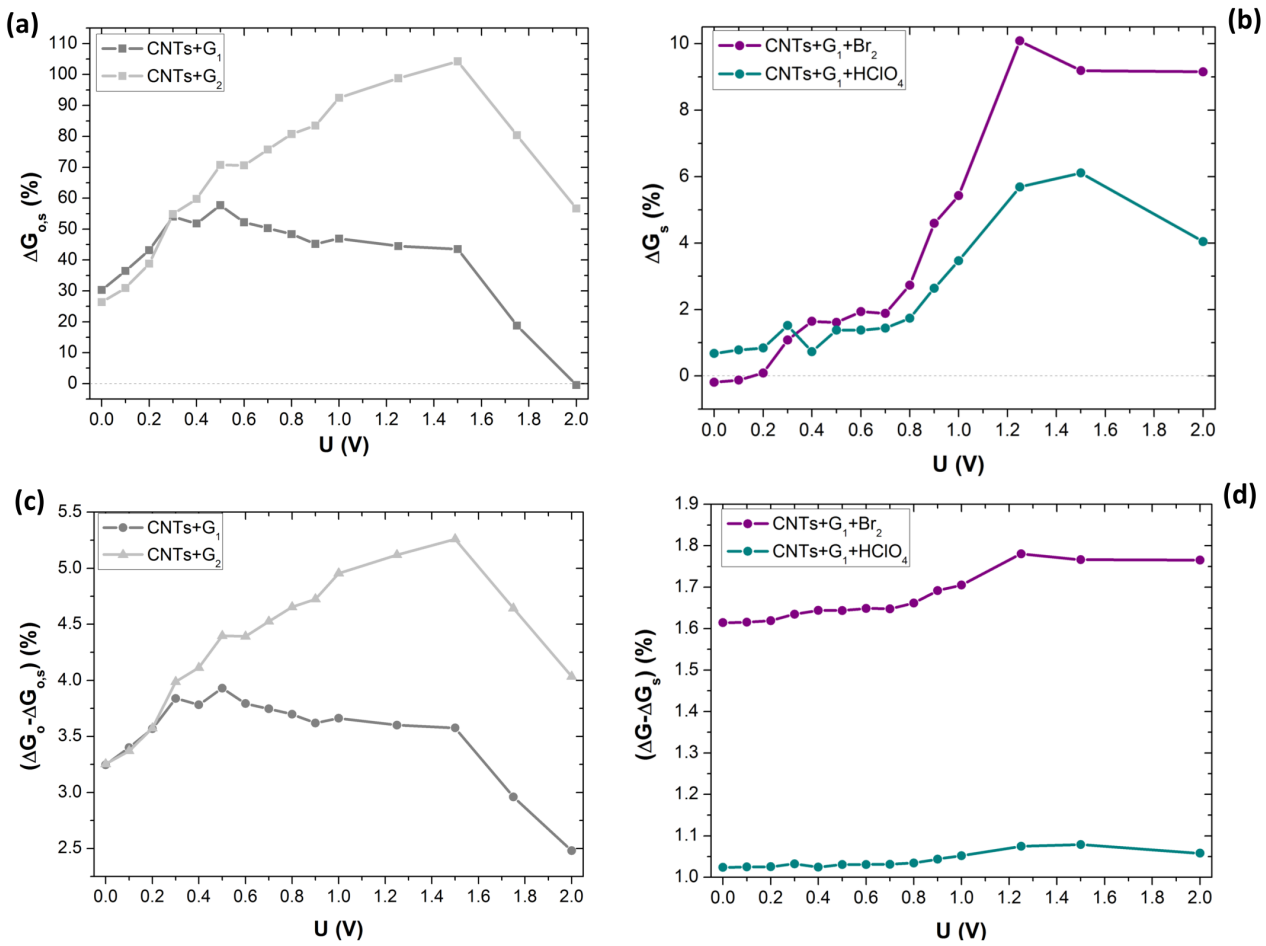

Figure S2: (a),(b) The computed specific conductance change with applied voltage. $\Delta \mathrm{G}_{s}$ is calculated as $\left(\mathrm{G}_{s, \text { doped }}-\mathrm{G}_{s, \text { pure }}\right) /\left(\mathrm{G}_{\text {s,pure }}\right) \times 100 \% . \Delta \mathrm{G}_{o}$ is calculated with respect to the device model containing only CNTs. (c), The difference between the conductance change and the specific conductance change obtained by subtraction of results from Fig. 11 (b) and Fig.S2 (a). (d) The difference between the conductance change and the specific conductance change obtained by subtraction of results from Fig. 11 (d) and Fig.S2 (b). 

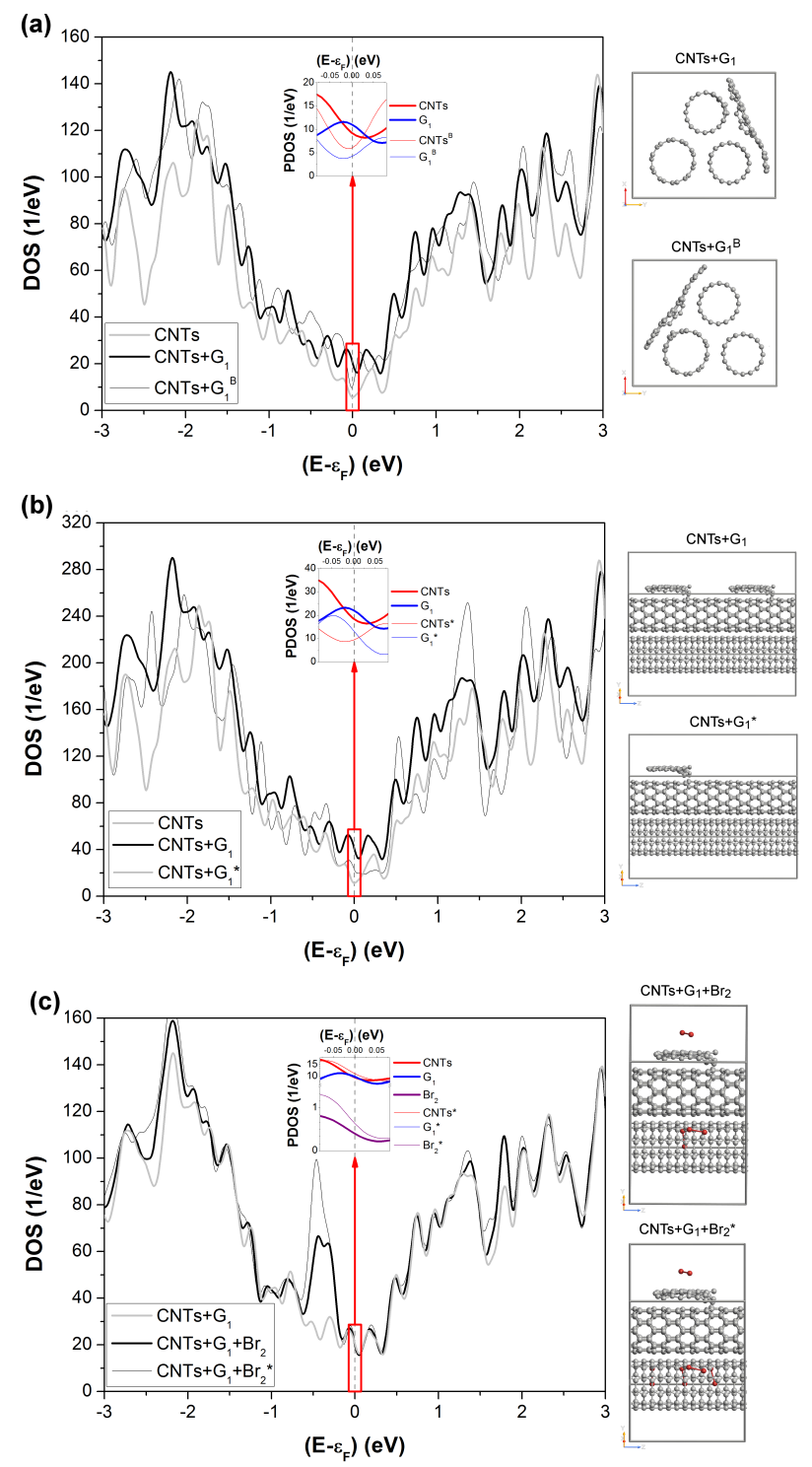

Figure S3: Left panel: The computed total density of states of $\mathrm{CNTs}+\mathrm{G}_{1}$ hybrid systems. The total density of pure CNT fibre is shown as a reference in panel (a) and (b), while the total density of undoped $\mathrm{CNTs}+\mathrm{G}_{1}$ system is added to the panel (c). Insets show the projected density of states. Right panel: Atomistic cross-sectional and side views of fully optimized systems. The $\mathrm{C}$ atoms are depicted in grey while $\mathrm{Br}$ atoms are shown in dark red. In the CNTs $+\mathrm{G}_{1}^{B}$ model, the graphene flake was placed above two different CNTs but the distance between neighbouring flakes remains the same. The concentration of graphene flakes and thus the distance between them has been reduced in $\mathrm{CNTs}_{\mathrm{T}} \mathrm{G}_{1}{ }^{*}$ model, while $\mathrm{CNT}+\mathrm{G}_{1}+\mathrm{Br}_{2}{ }^{*}$ contains two more $\mathrm{Br}_{2}$ molecules than was introduced in $\mathrm{CNT}+\mathrm{G}_{1}+\mathrm{Br}_{2}$ model 

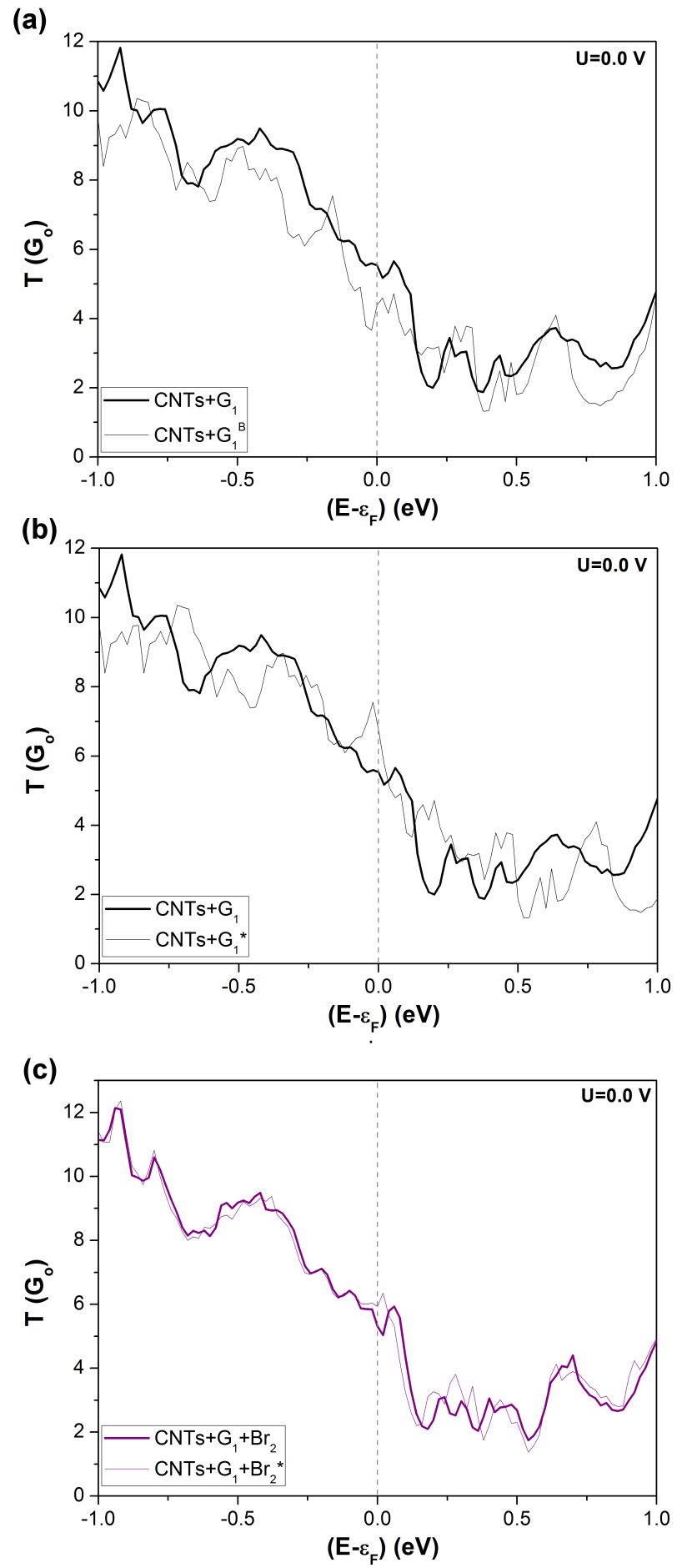

Figure S4: The zero-bias transmission spectra of $\mathrm{CNTs}+\mathrm{G}_{1}^{B}, \quad \mathrm{CNTs}+\mathrm{G}_{1}{ }^{*}$, and $\mathrm{CNTs}+\mathrm{G}_{1}+\mathrm{Br}_{2}{ }^{*}$ models. The zero-bias transmission spectra of $\mathrm{CNT}+\mathrm{G}_{1}$ hybrid system is shown as a reference in panel (a) and (b), while zero-bias transmission spectrum of $\mathrm{Br}_{2}$ doped $\mathrm{CNTs}+\mathrm{G}_{1}$ system is presented in panel (c). 
(a)

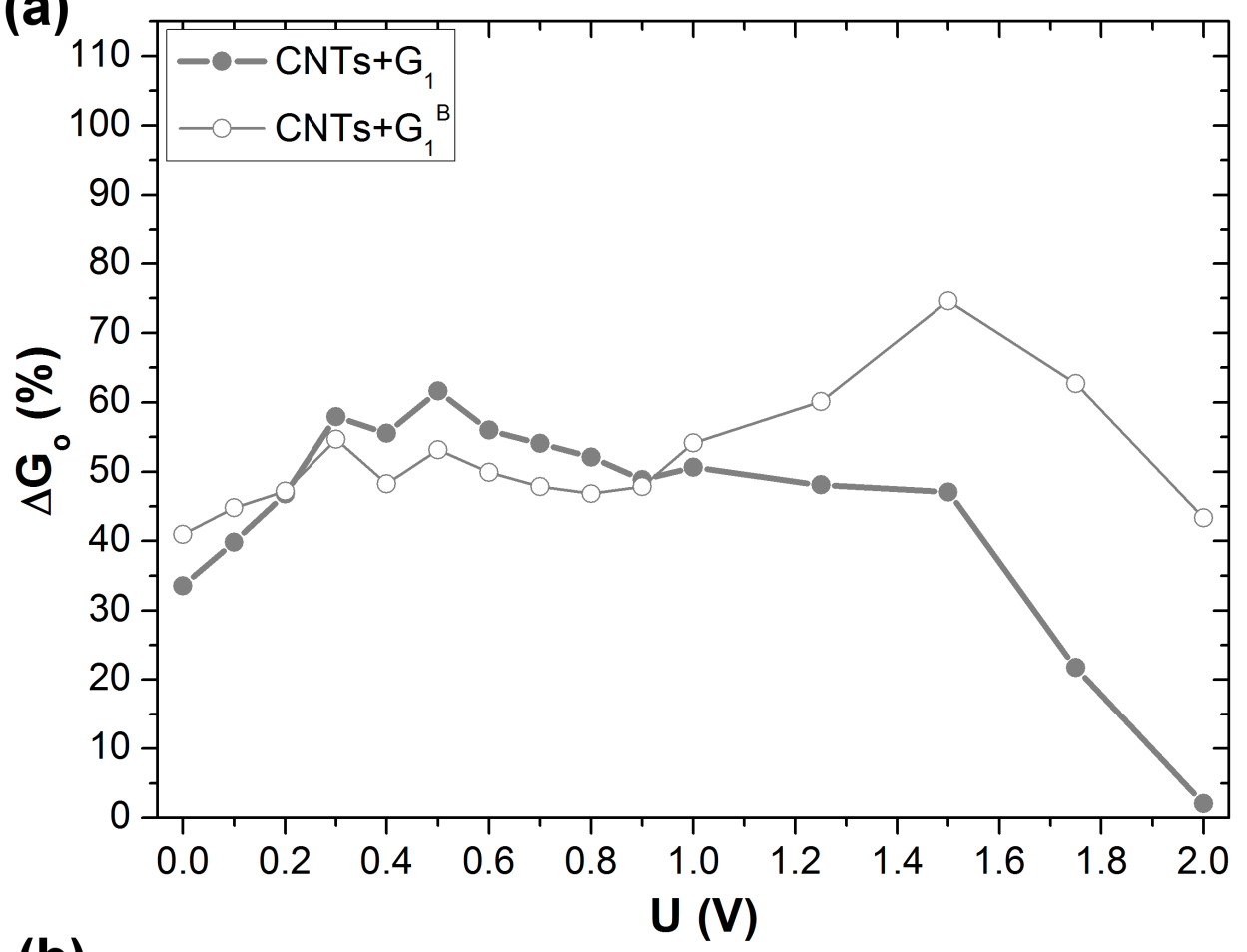

(b)

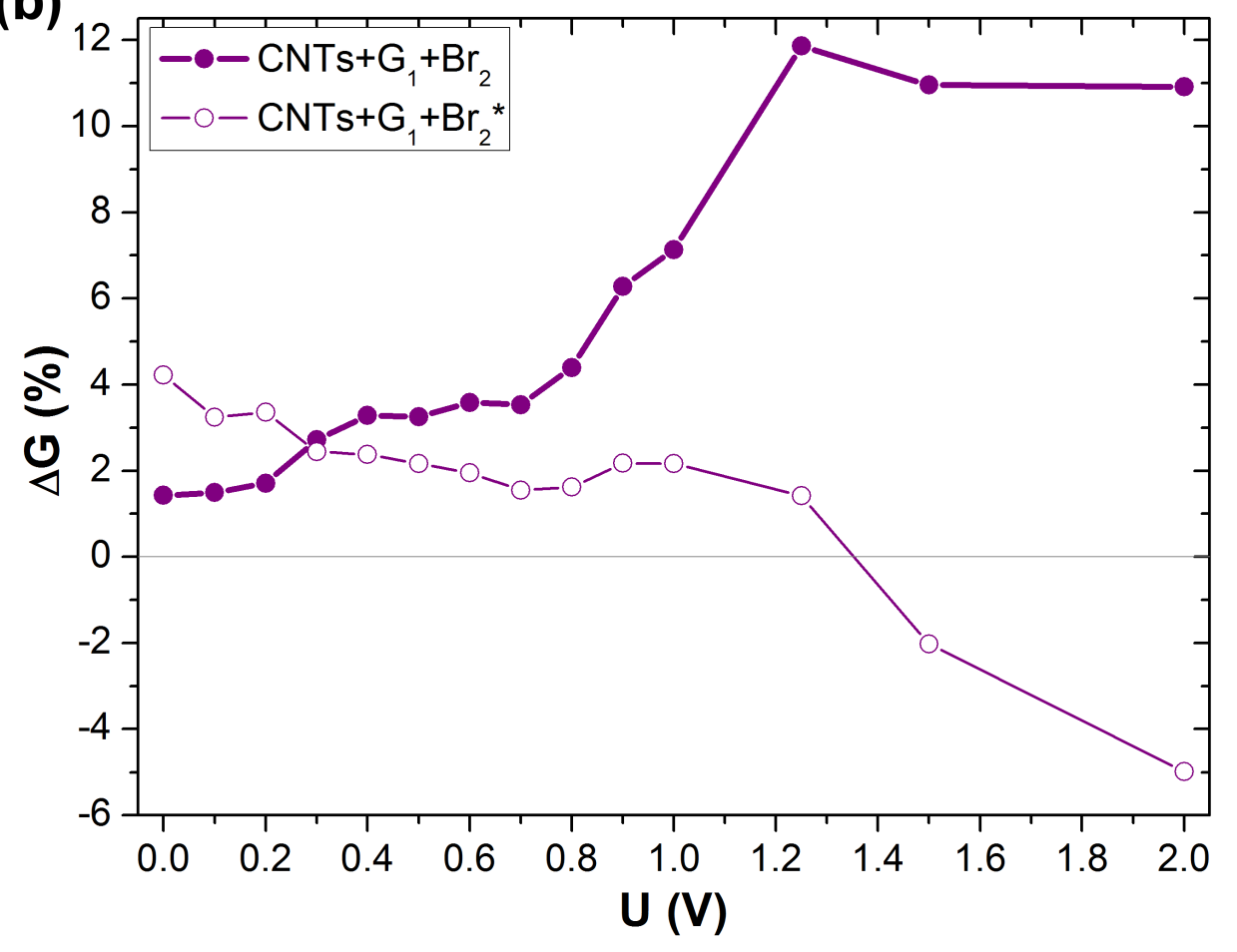

Figure S5: The computed conductance change of (a) CNTs $+\mathrm{G}_{1}^{B}$ and (b) CNTs $+\mathrm{G}_{1}+\mathrm{Br}_{2}{ }^{*}$ models as a function of applied voltage. 
Table S1: Coefficients of variation of nanotube radius (CV) of all three CNTs of all types of studied systems. CV of the device configurations are given in the brackets.

\begin{tabular}{|c|c|c|c|c|}
\hline system & doping & $\mathrm{CV}_{(5,5)}$ & $\mathbf{C V}_{(8,0)}^{I}$ & $\mathbf{C V}_{(8,0)}^{I I}$ \\
\hline CNTs & - & $\begin{array}{c}0.0128 \\
(0.0272)\end{array}$ & $\begin{array}{c}0.0117 \\
(0.0213)\end{array}$ & $\begin{array}{c}0.0086 \\
(0.0179)\end{array}$ \\
\hline \multirow{3}{*}{$\mathrm{CNTs}+\mathrm{G}_{1}$} & - & $\begin{array}{c}0.0128 \\
(0.0258)\end{array}$ & $\begin{array}{c}0.0431 \\
(0.0391)\end{array}$ & $\begin{array}{c}0.0114 \\
(0.0234)\end{array}$ \\
\hline & $\mathrm{Br}_{2}$ & $\begin{array}{c}0.0128 \\
(0.0258)\end{array}$ & $\begin{array}{c}0.0431 \\
(0.0391)\end{array}$ & $\begin{array}{c}0.0117 \\
(0.0234)\end{array}$ \\
\hline & $\mathrm{HClO}_{4}$ & $\begin{array}{c}0.0127 \\
(0.0258)\end{array}$ & $\begin{array}{c}0.0436 \\
(0.0396)\end{array}$ & $\begin{array}{c}0.0116 \\
(0.0233)\end{array}$ \\
\hline \multirow{3}{*}{$\mathrm{CNTs}+\mathrm{G}_{2}$} & - & $\begin{array}{c}0.0128 \\
(0.0258)\end{array}$ & $\begin{array}{c}0.0454 \\
(0.0497)\end{array}$ & $\begin{array}{c}0.0241 \\
(0.0295)\end{array}$ \\
\hline & $\mathrm{Br}_{2}$ & 0.0128 & 0.0448 & 0.0304 \\
\hline & $\mathrm{HClO}_{4}$ & 0.0127 & 0.0445 & 0.0310 \\
\hline
\end{tabular}

S3. SEM image of G3 graphene flakes hybridized with CNT film via spray coating technique at the magnification comparable to the magnifications of images of M25 and GNP flakes.

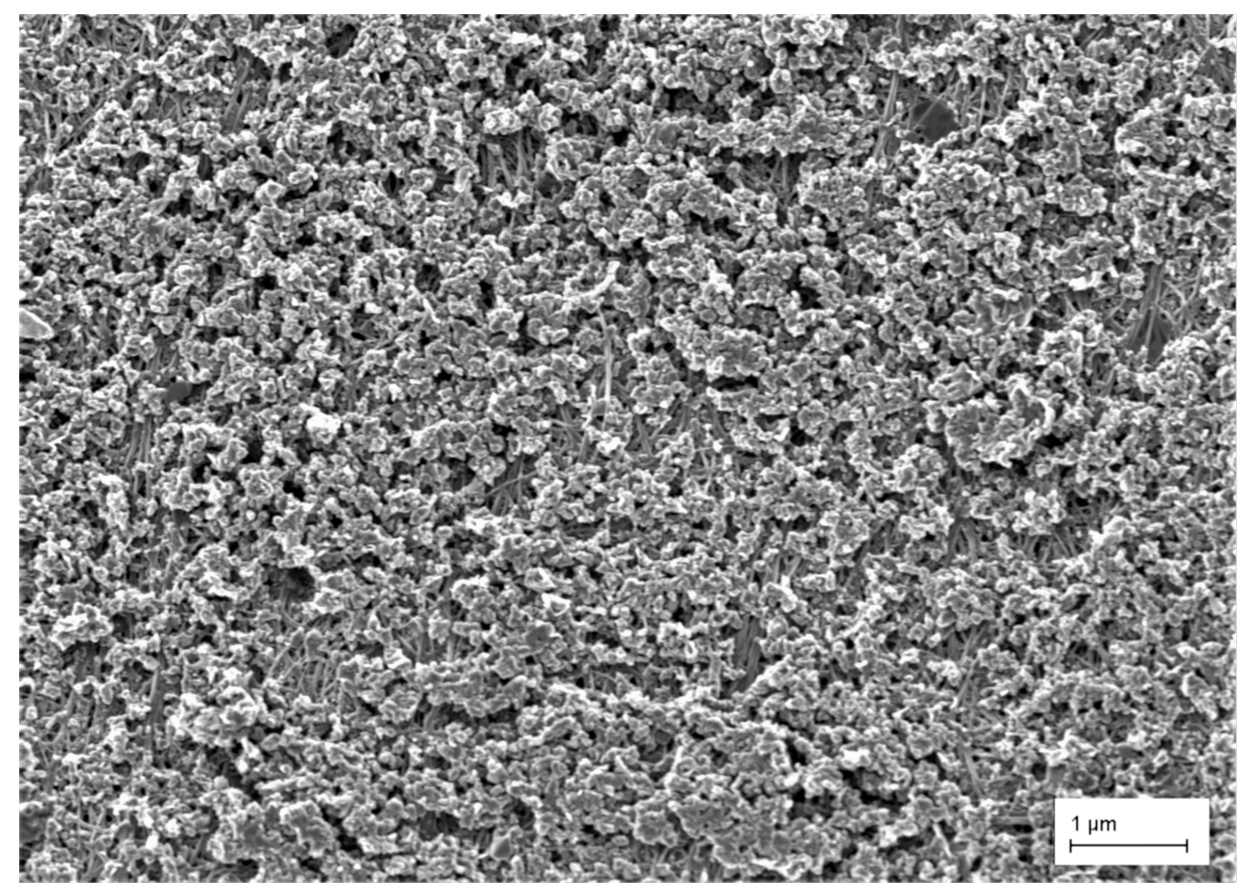

Figure S6: SEM image of G3 graphene flakes hybridized with CNT film via spray coating technique. Scale bar $1 \mu \mathrm{m}$. 


\section{References}

S1. Milowska, K. Z. Influence of Carboxylation on Structural and Mechanical Properties of Carbon Nanotubes: Composite Reinforcement and Toxicity Reduction Perspectives. J. Phys. Chem. C 2015, 119, 26734-26746.

S2. Datta, S. Electronic Transport in Mesoscopic Systems; Cambridge Studies in Semiconductor Physics and Microelectronic Engineering; Cambridge University Press, 1995. 